\title{
O CONHECIMENTO dOS FAMILIARES ACERCA DA PROBLEMÁtica do PORTADOR DE DIABETES MELLITUS ${ }^{1}$
}

\author{
Ana Emilia Pace ${ }^{2}$ \\ Polyana Duckur Nunes ${ }^{3}$ \\ Katia Ochoa-Vigo 4
}

Pace AE, Nunes PD, Ochoa-Vigo K. O conhecimento dos familiares acerca da problemática do portador de diabetes mellitus. Rev Latino-am Enfermagem 2003 maio-junho; 11(3):312-9.

Considerando a família como fonte de apoio ao doente, realizou-se este estudo visando analisar a problemática do portador de diabetes na visão dos familiares, em Unidade Ambulatorial de um hospital universitário do interior do Brasil. Os dados foram coletados de 24 familiares por meio de entrevista semi-estruturada. Para apresentação e análise dos dados, utilizou-se estatística descritiva. Nos resultados, observou-se que os familiares conhecem as causas do diabetes parcialmente. Quanto às condições de adesão ao tratamento, 70,8\% consideraram prioritário dieta adequada que, concomitantemente, foi salientada como problema. Em relação às doenças decorrentes, 23 salientaram problemas nos rins/visão. Quanto aos cuidados com os pés, destacou-se a utilização de meias de algodão e limpas. Referente às experiências dos familiares com pessoas portadoras de problemas nos pés, 83,3\% responderam afirmativamente, destacando as amputações/úlceras. Dados apontam necessidade de educação aos familiares, uma vez que proporcionam suporte no controle da doença e na prevenção de complicações.

DESCRITORES: diabetes mellitus, apoio familiar

\section{FAMILY KNOWLEDGE ABOUT THE PROBLEMATICS OF PATIENTS WITH DIABETES MELLITUS}

Considering the family as a source of support to the patient, this study aimed to analyze the problematics concerning people with diabetes in the opinion of relatives, in an outpatient unit of a university hospital in the interior of Brazil. Data were collected through semi-structured interviews with 24 family members. Descriptive statistics were used for data presentation and analysis. Results showed that the family members partially knew about the causes of diabetes. Regarding treatment adherence discussions, $70.8 \%$ considered an adequate diet a priority, which was concomitantly highlighted as a problem. Concerning related diseases, 23 persons pointed out kidney and vision problems. With respect to feet care, they emphasized the use of clean cotton socks. $83.3 \%$ had contact with patients with feet problems, mentioning amputations/ulcers. Data pointed out the need for educating family members, since they are a source of support in controlling the disease control and preventing complications.

DESCRIPTORS: diabetes mellitus, family support

\section{EL CONOCIMIENTO DE LOS FAMILIARES SOBRE LA PROBLEMÁtICA DEL PORTADOR DE DIABETES MELLITUS}

Considerando la familia como fuente de apoyo al enfermo, se realizó un estudio buscando analizar la problemática del portador de diabetes en la visón de los familiares en La consulta externa de un hospital universitario del interior del Brasil. Los datos fueron recolectados con 24 familiares por medio de entrevista semi-estructurada. Para la presentación y el análisis de los datos se utilizó la estadística descriptiva. En los resultados se observó que los familiares conocen parcialmente las causas de la diabetes. En relación con las condiciones de adhesión al tratamiento, $70,8 \%$ consideró prioritaria la dieta adecuada que, concomitantemente, fue destacada como problema. En relación con las enfermedades asociadas, 23 resaltaron problemas en riñones/vista. En referencia a los cuidados con los pies, se destacó la utilización de medias de algodón y limpias. EN lo que se refiere a las experiencias de los familiares con personas portadoras de problemas en los pies, 83,3\% respondieron afirmativamente, destacando las amputaciones/úlceras. Los datos muestran la necesidad de educación a los familiares, una vez que proporcionan soporte en el control de la enfermedad y en la prevención de complicaciones.

DESCRIPTORES: diabetes mellitus, apoyo familiar

\footnotetext{
${ }^{1}$ Este trabalho formou parte do Projeto "O Enfermeiro na prevenção e detecção precoce das complicações em extremidades de membros inferiores das pessoas portadoras de diabetes mellitus", tendo recebido apoio financeiro do Conselho Nacional de Pesquisa -CNPq, no período de março de 2000 a fevereiro de 2001; ${ }^{2}$ Professor Doutor, e-mail: aeferraz@eerp.usp.br; ${ }^{3}$ Graduanda; ${ }^{4}$ Doutoranda do Programa de Interunidades de Doutoramento em Enfermagem, e-mail: kovpe@latinmail.com. Escola de Enfermagem de Ribeirão Preto da Universidade de São Paulo, Centro Colaborador da OMS para o desenvolvimento da pesquisa em enfermagem
} 
INTRODUÇÃO

Odiabetes mellitus constitui, atualmente, um dos principais problemas de saúde, que se refere tanto ao número de pessoas afetadas, gerando incapacidade e mortalidade, quanto ao elevado investimento do governo para o controle e tratamento de suas complicações. Ele já é a quarta causa de morte no Brasil.

Estima-se que, no País, existem mais de cinco milhões de pessoas diabéticas, das quais cerca de $50 \%$ desconhecem o diagnóstico ${ }^{(1)}$. Sua prevalência, entre as pessoas com 30 a 69 anos que moram na região urbana, é de $7,6 \%{ }^{(2)}$.

Dessa maneira, cabe aos profissionais de saúde estar atentos na identificação das pessoas com risco para o diabetes mellitus e intensificar as ações para promover o seu controle, entre os já diagnosticados. Acredita-se que a família tem papel fundamental em ambas as situações.

Sabe-se que a família e os amigos influenciam no controle da doença quanto ao seguimento do tratamento, da dieta e na participação em um programa regular de exercícios. Um estudo demonstrou que as pessoas com diabetes mellitus, que tiveram apoio adequado de amigos e familiares, aderiram melhor às condutas de autocuidado. Os autores ainda relatam que avaliar os meios de apoio do paciente pode ajudar a identificar as suas necessidades de assistência, no propósito de evitar as complicações de longo prazo ${ }^{(3)}$.

Dessa forma, os profissionais de enfermagem, com o passar do tempo, têm reconhecido a importância do significado da família na saúde e bem-estar de cada um de seus membros, bem como também de sua influência sobre a doença ${ }^{(4)}$.

Nos últimos anos, trabalhos enfocando a família vêm sendo realizados de forma crescente, nas mais diversas áreas da enfermagem, buscando reconhecer 0 significado da doença à família, bem como a influência da doença de um membro sobre o bem-estar dos demais ${ }^{(5)}$.

A doença na família afeta tanto o relacionamento entre o casal, entre os irmãos, bem como as relações estabelecidas com o sistema de saúde. Estudo descreve o quanto a família é afetada pelas mudanças nos papéis, dinâmica familiar e aspectos econômicos ${ }^{(5)}$.

Concorda-se que a família constitui um sistema no qual o comportamento de cada um dos membros é interdependente ao dos outros. O grupo familiar pode ser visto como um conjunto que funciona como uma totalidade, em que as particularidades dos membros não bastam para explicar o comportamento de todos os outros membros. Desse modo, a análise de uma família não é a soma da análise de seus membros individuais ${ }^{(6)}$.

Assumindo a família como um sistema, fica claro que a experiência de cada um de seus membros afeta o sistema familiar, dado que o comportamento de cada pessoa afeta e é afetado pelo comportamento de cada uma das outras pessoas. Nessa perspectiva, a enfermagem deve focalizar interações entre seus membros, bem como as interações com os outros subsistemas (profissionais de saúde, parentes, amigos) ao invés de estudar só a pessoa doente ${ }^{(5)}$.

Considerando que os familiares podem contribuir para o cuidado do paciente com doenças crônicas e, dessa maneira, prevenir e/ou diminuir as complicações dela decorrentes, realizou-se o presente estudo com o objetivo de analisar a problemática do portador de diabetes mellitus na visão dos membros familiares; qual a influência da doença na dinâmica familiar e quanto sabem sobre a patologia.

\section{MÉTODOS}

O presente estudo é de natureza descritiva e foi realizado no Ambulatório de Endocrinologia e Metabologia do Hospital das Clínicas da Faculdade de Medicina de Ribeirão Preto, da Universidade de São Paulo (HCFMRPUSP), cuja população esteve composta pelos familiares acompanhantes das pessoas com diagnóstico de diabetes mellitus atendidas nessa unidade. Foram considerados alguns critérios de inclusão para a seleção da amostra do estudo: ser familiar acompanhante da pessoa com diabetes mellitus, conhecer a doença do familiar há pelo menos um ano, possuir idade superior a 21 anos, manter bom senso e raciocínio e concordar em participar do estudo, manifestando sua aquiescência por escrito. Desse modo, a amostra foi constituída por 24 familiares dos pacientes portadores de diabetes, que compareceram aos retornos ambulatoriais, no período de abril de 2000 a março de 2001.

Os dados foram coletados todas as segundasfeiras, por meio de um instrumento semi estruturado aplicado por um entrevistador previamente treinado. $\mathrm{O}$ instrumento era composto por: dados sociodemográficos, 
conhecimentos sobre o diabetes mellitus, tratamento, dificuldades, complicações e cuidados com os pés.

A entrevista foi realizada no Ambulatório de Endocrinologia e Metabologia do HCFMRP-USP, após a consulta médica agendada pelo portador de diabetes mellitus, a qual requeria de 20 a 30 minutos para sua total aplicação.

Para a organização dos dados, utilizou-se o Programa Microsoft Acces 97, no propósito de obter-se agilidade na interpretação e análise das informações coletadas nas entrevistas.

A análise dos dados foi feita no Programa Epinfo, versão 97, por meio de testes estatísticos descritivos (média, percentuais e freqüências). Outros dados quantitativos foram categorizados de acordo com a importância e apresentados em freqüências.

\section{ANÁLISE E DISCUSSÃO DOS RESULTADOS}

Inicialmente, destaca-se que, dos 24 familiares que compuseram a amostra, dezenove eram pertencentes ao sexo feminino $(79,2 \%)$, e cinco, ao masculino $(20,8 \%)$ com idades entre 27 e 83 anos, sendo $45,8 \%$, entre 51 a 60 anos, tendo-se obtido uma média de 53,9 anos.

Dados relacionados com o grau de parentesco mostraram que doze (50\%) deles mantinham condição marital com o paciente, sendo que $50 \%$ dos entrevistados pertenciam à região de Ribeirão Preto e, entre eles, seis moravam na própria cidade.

Quanto ao nível de escolaridade, grande parte $(54,1 \%)$ possuía primeiro grau incompleto ou apenas foram alfabetizados; entretanto, 4,2\% dos entrevistados estiveram na condição de analfabetos. Em relação à ocupação, 37,5\% deles desempenhavam atividades do lar, e $29,2 \%$ estiveram na condição de aposentados.
Entre os dados acima referidos, percebe-se que mais de $50 \%$ da amostra encontrava-se em condições desvantajosas para assimilar os ensinamentos oferecidos pelos profissionais de saúde quanto às atividades de autocuidado do paciente (dieta, exercícios físicos e administração de medicamentos) no ambiente familiar. Essa situação pode ser importante, ao considerar a idade e o nível de escolaridade da amostra, sobretudo quando o paciente e acompanhante não compartilham o lar com outros membros da família que, mesmo não tendo comparecido ao local de saúde, oferecem suporte neste cuidado, caso ele esteja ciente da doença e seus riscos para com a saúde.

Não foram encontrados, na literatura, estudos que apresentassem as condições relatadas até aqui, apesar de que conhecer as caraterísticas dos familiares faz-se importante para saber de que tipo de apoio o paciente dispõe no domicílio e de que forma contribui ou reforça as atividades de seu autocuidado. No entanto, é necessário aprofundar esse tema, pois um membro da família poderia não ser suficiente para avaliar o contexto familiar, mas, sim, cada um de seus membros, tendo em vista que, entre eles, cada um deles exerce influência no modo de cuidar da saúde e da doença.

Por meio da Tabela 1, apresenta-se a opinião dos 24 familiares sobre o que é o diabetes mellitus e quais as suas causas, sendo que foram consideradas, para cada uma delas, quatro categorias de respostas, conforme foi descrito.

Salienta-se que as respostas foram categorizadas considerando que, pelo menos, dois critérios descritos na categoria "Correta", e um, na "Parcial", foram expressos pelos familiares. Para as outras categorias não foram incluídas opções. No entanto, qualquer resposta errada ou vaga em relação ao diabetes adequou-se entre elas. $A$ seguir, apresenta-se a tabela referida.

Tabela 1 - Distribuição dos familiares de pacientes diabéticos, segundo as conceituações e causas do diabetes mellitus. Ribeirão Preto, 2001

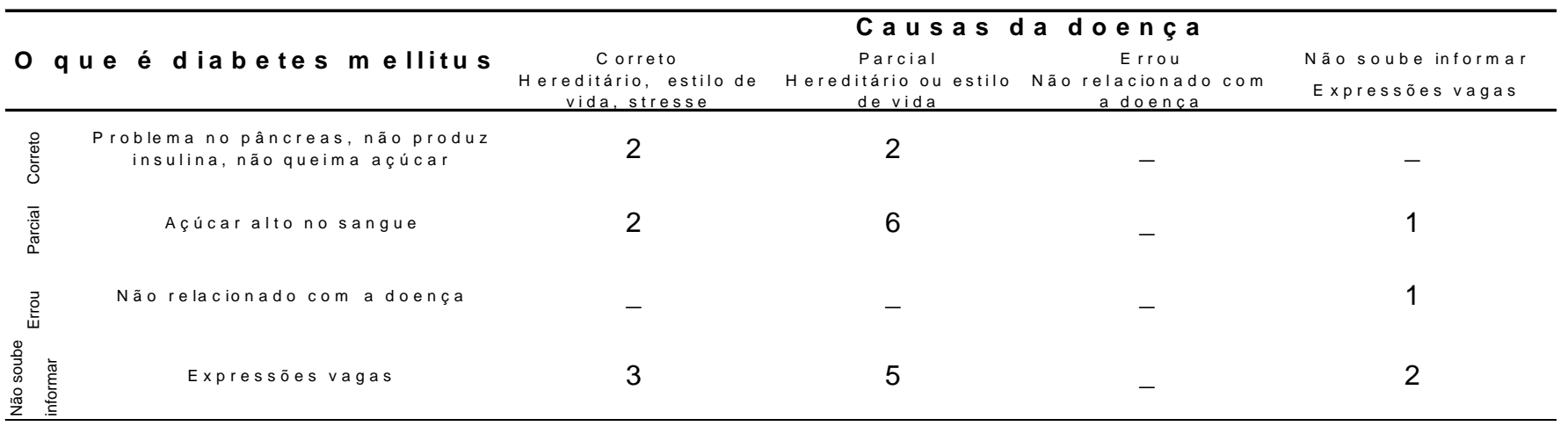


Considerando distintamente as perguntas, destacam-se, na questão "o que é diabetes", nove respostas na categoria "Parcial" e dez na categoria "Não soube informar". Na questão sobre "Causas da doença", treze respostas foram classificadas na categoria "Parcial", e sete, na "Correta".

Ao analisar as duas questões conjuntamente, observam-se apenas duas respostas para a categoria "Correta", seis para a "Parcial" e cinco entre as categorias "Parcial" e "Não soube informar". Pode-se identificar, também, que a maioria dos familiares (20) estão mais bem informados quanto às causas da doença em detrimento ao o que é diabetes mellitus (12).

Nos resultados, identifica-se que os familiares entrevistados possuem poucas informações sobre o diabetes. Esta situação é agravada pelas informações erradas e vagas encontradas no estudo como: uma amiga, fome, tontura, dentre outros.

Desta forma, entende-se que as ações de caráter preventivo e de controle da doença ficam prejudicadas, pois, como prevenir e assumir o cuidado, se se desconhece o que realmente é a doença e quais suas causas?

A Tabela 2 ilustra os resultados do tratamento seguido pelo paciente diabético na opinião do familiar. Observa-se que dezessete $(70,8 \%)$ referiram que o paciente segue uma dieta adequada e apenas oito $(33,3 \%)$ praticam atividades físicas regularmente.

Tabela 2 - Distribuição dos acompanhantes de pacientes diabéticos, segundo a adesão ao tratamento do diabetes pelo familiar e as condições de adesão. Ribeirão Preto, 2001

\begin{tabular}{|c|c|c|c|c|c|c|c|c|}
\hline \multirow{3}{*}{$\begin{array}{l}\text { Adesão ao tratamento do } \\
\text { diabetes pelo familiar }\end{array}$} & \multicolumn{6}{|c|}{ Condições de adesão } & & \\
\hline & \multicolumn{2}{|c|}{ Sim } & \multicolumn{2}{|c|}{ Não } & \multicolumn{2}{|c|}{ As vezes } & \multicolumn{2}{|c|}{ TOTAL } \\
\hline & № & $\%$ & № & $\%$ & № & $\%$ & № & $\%$ \\
\hline Dieta adequada & 17 & 70,8 & 3 & 12,5 & 4 & 16,7 & 24 & 100 \\
\hline Desenvolvimento de atividades físicas & 8 & 33,3 & 2 & 8,3 & 14 & 58,3 & 24 & 100 \\
\hline Consumo de remédio caseiro (chá) ${ }^{*}$ & 2 & 8,3 & 1 & 4,2 & 21 & 87,5 & 24 & 100 \\
\hline Administração de insulina & 13 & 54,2 & - & - & 11 & 45,8 & 24 & 100 \\
\hline Administração de antidiabético oral & 15 & 62,5 & - & - & 9 & 37,5 & 24 & 100 \\
\hline
\end{tabular}

Carquejo, cidreira, planta insulina*

Pelos dados da Tabela 2, verifica-se que grande parte dos familiares têm consenso sobre o tratamento do paciente, o que é considerado positivo, pois esse comportamento pode ser um fator facilitador para a adesão ao tratamento.

O seguimento da dieta e a prática de atividades físicas compõem atividades fundamentais para o tratamento do diabetes. O não seguimento da dieta e o fato de "Às vezes" a seguirem podem significar ingesta inadequada de nutrientes o que contribuiria com 0 descontrole metabólico.

As atividades ou exercícios físicos, como as caminhadas, parecem não ser relevantes para a população diabética, isso porque até os mesmos familiares assim o vêm, observando cada doente, sendo que $58,3 \%$ deles não os realizam, resultado semelhante ao identificado em uma Unidade Básica e Distrital da Secretária Municipal de Saúde de Ribeirão Preto ${ }^{(7)}$.

Sabe-se que a realização de atividades físicas contribui para queimar açúcar no sangue, melhorar a circulação cardíaca e periférica no organismo, bem como para fortalecer e nutrir os tecidos, dentre outros, além de melhorar a disposição e sensação de bem-estar do paciente $^{(1,8)}$. No entanto, tais atividades devem ser realizadas após uma avaliação médica minuciosa, que discrimine as condições macrovasculares e microvasculares ${ }^{(1)}$. Salienta-se ainda que o programa de exercícios sempre deve ser feito sob orientação e controle da equipe de saúde, face aos prováveis riscos que poderiam acometer os membros inferiores (bolhas, calos, rachaduras, fissuras, dentre outros) pela freqüente perda de sensibilidade, originados pelas neuropatias periféricas e autonômicas ${ }^{(9)}$.

Quanto à dificuldade dos pacientes no tratamento do diabetes mellitus, identificou-se que quatorze $(58,3 \%)$ dos vinte e quatro familiares apontaram a dieta, e uma porcentagem significativa $(29,2 \%)$ indicou o fator financeiro.

Verifica-se que existe uma relação entre as duas dificuldades, visto que uma melhor renda poderia facilitar a aquisição de alimentos, especialmente se visualizamos que, na Tabela 2, a maioria dos acompanhantes $(70,8 \%)$ referiram que a adesão ao tratamento pelo paciente relaciona-se à dieta adequada. Dessa forma, a educação alimentar necessita ser difundida para que todos tenham 
conhecimento sobre alimentos de boa qualidade e de baixo custo.

Alguns dos familiares referiram que os pacientes "Às vezes" têm dificuldade em aceitar a doença (16,7\%) e que ainda $12,5 \%$ deles têm dificuldade em se aplicar a insulina. Isso pode causar algum grau de estresse e desconforto, porém a resignação à doença e seu tratamento são importantes para melhor controle. No entanto, destaca-se que a fase de não aceitação é mais comum nos períodos iniciais da descoberta da doença que, provavelmente, inclui não só os pacientes, mas também todo o grupo familiar, o que estaria associado a um pobre controle da glicemia sangüínea ${ }^{(7)}$.

Em relação à administração da insulina, pode-se supor que a dificuldade está relacionada à falta de informação e treinamento adequado dos pacientes para a auto-aplicação, e alguns deles até podem precisar de auxílio nessa tarefa ${ }^{(7)}$, devido às próprias limitações. É provável que a dificuldade esteja também associada às eventuais complicações locais e injúrias que essa prática pode ocasionar ao paciente, devido à freqüente reutilização das seringas descartáveis ${ }^{(10)}$.

Quanto à presença ou não de mudanças no diaa-dia da família, observou-se que doze (50\%) dos entrevistados referiram ter havido mudanças, dentro dos quais foram ressaltados, novamente, aspectos relacionados aos hábitos alimentares $(20,8 \%)$ e às dificuldades econômicas (12,5\%). Tais dados demonstram, uma vez mais, quão determinantes podem ser, no contexto da dinâmica familiar, as questões financeiras e alimentares. Situação que vem corroborar o grande impacto que a doença tem nos hábitos alimentares da família que, muitas vezes, está restringida às possibilidades econômicas e aos fatores culturais e psicológicos $^{(7)}$.

Considerando que a dieta constitui um dos principais meios para o êxito do tratamento do diabetes, é importante identificar, na amostra estudada, que pelo menos $50 \%$ dos entrevistados reconheceram que a família toda experimentou algum grau de mudança nesse aspecto. Isso se deve ao fato de que $29,2 \%$ dessa população encontra-se na condição de aposentado (a), obrigando a família a adaptar-se a um novo padrão alimentar, o que pode acarretar outro fator dificultador para o tratamento da doença.

Outros problemas de saúde podem ser ocasionados pelo diabetes mellitus, tais como doenças renais, visuais, cardíacas, vasculares e neuropáticas; vinte e três familiares responderam que, geralmente, ocorre algum tipo de problema associado, ou seja, 12 responderam de forma "Correta" e 11 de forma "Parcial".

Estudos demonstram que o diabetes mellitus produz, concomitantemente, alterações em vários aparelhos e sistemas mais freqüente nos diabéticos não controlados, que são suscetíveis a complicações altamente incapacitantes como cegueira, insuficiência renal crônica dependente ou não de hemodiálise e doenças cardiovasculares, que causam grande dificuldade para a realização diária das atividades produtivas ${ }^{(2,11)}$. Os resultados apontam esta realidade, sendo que doze dos familiares estão cientes dos outros problemas que os pacientes poderiam vir a ter no decorrer de sua vida. No entanto, é preciso reforçar a importância do controle metabólico para prevenir ou retardar o aparecimento das complicações crônicas do diabetes e assim favorecer a qualidade de vida de pacientes e familiares.

Ao serem questionados sobre se o diabetes poderia ocasionar algum tipo de problema nos pés, vinte e um $(87,5 \%)$ responderam afirmativamente, destacando as amputações, úlceras, micoses, queimação, falta de cicatrização das feridas e infecção.

A literatura destaca o risco de os pacientes desenvolverem, especialmente, o chamado "pé diabético", caracterizado por lesões que ocorrem como conseqüência de neuropatias, em $90 \%$ dos casos, doença vascular e deformidades, lesões geralmente produzidas por trauma que, freqüentemente, complicam-se com infecção, podendo terminar em amputação, se não for instituído um tratamento precoce e adequado ${ }^{(12)}$. Uma amputação, com freqüência, está precedida de problema neuropático que se manifesta, inicialmente, com o aparecimento de calos e feridas nas plantas dos pés como conseqüência do atrito e da pressão excessiva, em certas áreas, pela adoção inadequada do pisar ${ }^{(8)}$.

Os problemas neuropáticos também produzem, simultaneamente, dor, sob forma de queimação e ardor, diminuição da sensibilidade, fraqueza nas pernas e pés, junto a uma ausência dos reflexos do joelho e pés ${ }^{(8-9)}$. Esses sintomas foram referidos por grande parte dos familiares, seja de forma isolada ou associada, comprometendo-os a estar atentos à identificação precoce desses sinais que podem conduzir à perda do membro inferior.

Os dados relacionados com os pés, quanto à utilização de sapatos, meias e quanto aos cuidados, estão distribuídos nas Tabelas 3 e 4, que serão analisados, de maneira paralela, por tratar-se de assuntos complementares. 
Tabela 3 - Distribuição dos familiares de pacientes diabéticos, segundo os cuidados com sapatos e meias e as atividades realizadas. Ribeirão Preto, 2001

\begin{tabular}{|c|c|c|c|c|c|c|c|}
\hline \multirow{3}{*}{\multicolumn{2}{|c|}{ Atividades realizadas }} & \multicolumn{6}{|c|}{ Cuidado com sapatos e meias } \\
\hline & & \multicolumn{2}{|c|}{ Ao comprar os sapatos } & \multicolumn{2}{|c|}{ Ao calçar os sapatos } & \multicolumn{2}{|c|}{ No uso da meias } \\
\hline & & Sim & Não sabe & Sim & Não sabe & Sim & Não sabe \\
\hline \multirow{3}{*}{ 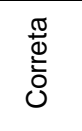 } & Macio e folgado & \multirow{3}{*}{8} & \multirow{3}{*}{-} & \multirow{3}{*}{8} & \multirow{3}{*}{-} & \multirow{3}{*}{4} & \multirow{3}{*}{-} \\
\hline & Olhar dentro antes de calçar & & & & & & \\
\hline & feitas de algodão e no avesso & & & & & & \\
\hline \multirow{3}{*}{$\begin{array}{l}\frac{\bar{\sigma}}{0} \\
\frac{\pi}{\pi} \\
0\end{array}$} & Macio ou folgado & \multirow{3}{*}{8} & \multirow{3}{*}{-} & \multirow{3}{*}{5} & \multirow{3}{*}{-} & \multirow{3}{*}{10} & \multirow{3}{*}{-} \\
\hline & calçar devagar, limpar por fora.. & & & & & & \\
\hline & algodão ou limpa & & & & & & \\
\hline \multirow{3}{*}{ 른 } & Justo e/ou de bico & \multirow{3}{*}{5} & \multirow{3}{*}{-} & \multirow{3}{*}{3} & \multirow{3}{*}{-} & \multirow{3}{*}{5} & \multirow{3}{*}{1} \\
\hline & Nenhum cuidado & & & & & & \\
\hline & feita de outro material & & & & & & \\
\hline \multirow{3}{*}{ 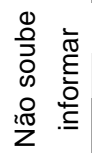 } & Expressões vagas & \multirow{3}{*}{-} & \multirow{3}{*}{3} & \multirow{3}{*}{3} & \multirow{3}{*}{5} & \multirow{3}{*}{ - } & \multirow{3}{*}{5} \\
\hline & Expressões vagas & & & & & & \\
\hline & Expressões vagas & & & & & & \\
\hline
\end{tabular}

Tabela 4 - Distribuição dos familiares de pacientes diabéticos, segundo cuidado com os pés e as atividades realizadas. Ribeirão Preto, 2001

\begin{tabular}{|c|c|c|c|c|c|}
\hline & \multirow{3}{*}{ Atividades realizadas } & \multicolumn{4}{|c|}{ Cuidado dos pés } \\
\hline & & \multicolumn{2}{|c|}{ No corte da unhas } & \multicolumn{2}{|c|}{$\mathrm{Na}$ presença de calos } \\
\hline & & Sim & Não sabe & Sim & Não sabe \\
\hline \multirow{2}{*}{$\begin{array}{l}\frac{\pi}{0} \\
\stackrel{0}{\circ} \\
0 \\
0\end{array}$} & Corte reto e não muito curto & \multirow{2}{*}{9} & \multirow[t]{2}{*}{ - } & \multirow{2}{*}{3} & \multirow[b]{2}{*}{-} \\
\hline & Pedra pome, lixa de papel, uso de palmilha & & & & \\
\hline \multirow{2}{*}{$\begin{array}{l}\frac{\bar{\pi}}{\overline{0}} \\
\frac{\pi}{\square}\end{array}$} & Corta aredondado, alicate desinfetado & \multirow{2}{*}{13} & \multirow{2}{*}{-} & \multirow{2}{*}{5} & \multirow[b]{2}{*}{-} \\
\hline & Amolhecimento dos calos & & & & \\
\hline \multirow{2}{*}{ כે } & Corte dos cantos e muito curto & \multirow[b]{2}{*}{-} & \multirow[b]{2}{*}{-} & \multirow{2}{*}{3} & \multirow[b]{2}{*}{-} \\
\hline & Uso de gilete, lixa de metal, deixar pés de molho & & & & \\
\hline \multirow{2}{*}{ 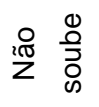 } & Expressões vagas & \multirow{2}{*}{2} & \multirow{2}{*}{-} & \multirow{2}{*}{-} & \multirow{2}{*}{1} \\
\hline & Expressões vagas & & & & \\
\hline
\end{tabular}

$\mathrm{Na}$ Tabela 3, observa-se que os dados referentes aos cuidados que se deve ter ao comprar sapatos estão contidos nos quadros brancos, onde se observa que oito familiares consideraram que devem ser macios e folgados, como critérios importantes. Verificou-se, também, a mesma freqüência (oito) para a condição "Parcial", nos quadros pontilhados.

Quando questionados sobre o que era mais importante ao calçar os sapatos (quadros pontilhados), oito apontaram que examiná-los era fundamental, devido à possibilidade de haver objetos que poderiam lesar os pés. Quanto aos cuidados com as meias (quadros cinza), dez mencionaram que deviam ser de algodão e sempre limpas. No estudo, foi considerado que, além de ser feitas de algodão, deveriam ser calçadas do avesso, pois as linhas da costura também poderiam ocasionar lesões.
Em relação aos cuidados com os pés, apresentados na Tabela 4, os dados referentes ao corte das unhas (quadros pontilhados) apontam que grande parte dos familiares considerou-os importante. Treze das respostas classificadas como "Parcial" indicaram corte arredondado e uso de alicate desinfetado, enquanto nove referiram que o corte deve ser reto e não muito rente, enquadrando-se na categoria "Correta".

Quanto aos cuidados com os calos (quadros cinza), segundo referência dos familiares, doze deles nunca apresentaram o problema, sendo que cinco cuidavam disso de forma "Parcial". Entretanto, apenas três pacientes trataram-nos de forma "Correta", ou seja, fizeram uso de pedra pomes, lixa de papel e palmilha, cuidados considerados fundamentais para se evitarem maiores ferimentos. 
Sapatos macios e folgados previnem o aperto e o ferimento dos pés, pois os problemas ocasionados pela neuropatia periférica e arteriopatia poderiam ser agravados. A utilização de sapatos justos e apertados pode contribuir para o aparecimento de calos, pela compressão repetida e contínua em determinada região dos pés. Para tanto, é importante não seccionar os calos com instrumentos cortantes, pois podem permitir o acesso de bactérias que provocariam infeções graves. Em relação ao uso de pedra pomes, ela pode ser aplicada, após o banho, com água morna, para remover os calos, de forma superficial e suave ${ }^{(8)}$.

As palmilhas também oferecem benefícios, especialmente porque aliviam a pressão em determinados pontos de forte compressão nos pés. Em relação ao corte das unhas, deve ser feito sempre procurando seguir linha reta, evitando tirar as cutículas, pois elas formam uma camada de proteção da pele, e a sua retirada, freqüentemente, pode ocasionar lesões que seriam porta de entrada para qualquer processo infeccioso ${ }^{(8)}$.

Considerando os benefícios que as meias de algodão proporcionam aos pacientes, ressalta-se que, de preferência, devem ser de cor branca, para poder identificar facilmente as sujidades e as secreções, que poderiam ser causadas por algum processo supurativo. Esse tipo de meia também proporciona adequada ventilação aos pés, ao contrário das de tecido sintético, que retêm o suor, por formar uma capa impermeável.

Finalmente, destacamos as respostas emitidas pelos familiares quanto a um contato prévio com pacientes que apresentaram problemas nos pés/pernas. Vinte $(83,3 \%)$ afirmaram conhecer, referindo-se às amputações $(41,7 \%)$, problemas de úlceras $(29,2 \%)$, problemas de infecção/amputação (8,3\%) e rachaduras (4,2\%).

Autores norte-americanos relatam que o diabetes é responsável por mais do $50 \%$ das amputações não traumáticas de membros inferiores ${ }^{(13-14)}$, e esse risco é quinze vezes maior do que em indivíduos não diabéticos ${ }^{(9)}$.

O problema mais freqüente, que precede às amputações, está determinado por úlceras acompanhadas geralmente de gangrena e infecção, demonstrando que, na maioria dos defeitos mutilantes, a combinação de isquemia e infecção é preponderante ${ }^{(9)}$. Desse modo, as úlceras, nos pés dos diabéticos, constituem a causa principal das amputações dos membros inferiores, ressaltando que é uma complicação extremamente comum entre essa população, chegando a ser a maior causa de hospitalização, morbidade e mortalidade ${ }^{(15)}$.

Dessa forma, evidencia-se, no estudo, que nossa população diabética apresenta as mesmas características relatadas na literatura, sendo que os dois primeiros problemas descritos nos resultados assim o confirmam.

Devido às repercussões mutilantes do diabetes mellitus, é importante promover uma adequada adesão ao tratamento por parte dos pacientes, a qual poderá ser grandemente favorecida com uma participação efetiva dos familiares. Essa atividade obterá sucesso se os profissionais de saúde estabelecerem um vínculo entre eles e os pacientes, fato que deve vir a reforçar investimentos nas atividades de ensino para com o paciente e sua família, entendendo que os membros que constituem o grupo familiar influenciam e/ou apóiam, de forma determinada, o comportamento desenvolvido pelos pacientes no seu autocuidado.

\section{CONCLUSÕES}

Ao observar as informações que os familiares referiram sobre o diabetes mellitus, identificou-se que uma grande parte dos entrevistados referiu conhecer quais são as causas do diabetes mellitus de forma "Parcial", sendo que só sete estiveram na categoria de resposta "Correta". Quanto ao tratamento do diabético, $70,8 \%$ referiram que o paciente segue uma dieta adequada, no entanto esta se constituía em uma das principais dificuldades que enfrentam os pacientes para tal adesão. Considerando os problemas de saúde decorrentes do diabetes, 23 familiares referiram que pode ocasionar, principalmente, doenças nos rins ou na visão.

A respeito dos cuidados com os pés e suas complicações, oito familiares apontaram que, na compra dos sapatos, deve ser observado que os mesmos sejam macios e folgados, sendo que igual número ressaltou a importância de inspecioná-los antes de serem calçados. Já nos cuidados dos pés, treze citaram que as unhas poderiam ser cortadas de forma arredondada e com alicate desinfetado, resposta categorizada como "Parcial". Quanto ao contato prévio dos entrevistados com pacientes que apresentaram problemas nos pés, 83,3\% deram resposta afirmativa, sendo mais freqüentes as amputações $(41,7 \%)$ e as úlceras (29,2\%).

Apesar do número reduzido da amostra, os dados apontam que os familiares possuem conhecimento, de forma parcial, sobre o diabetes mellitus e seus cuidados, reforçando a necessidade de ações educativas nessa área, uma vez que a família é considerada um fator de apoio fundamental na adesão do paciente ao tratamento, e pelo seu autocuidado. 


\section{REFERÊNCIAS BIBLIOGRÁFICAS}

1. American Diabetes Association Diabetes mellitus and exercise. Diabetes Care 2000; 23 (Suppl 1): 50-4.

2. Franco LA, coordenador. Estudo multicentrico sobre a prevalência do diabetes mellitus no Brasil. Informe Epidemiol SUS 1992; 1(3):47-73.

3. Wang C, Fenske MM. Self-care of the adults with non-insulindependent diabetes mellitus: influence of family and frieds. Diabetes Educ 1996; 22(5):465-70.

4. Wright LM, Leahey M. Nurses and Families: a guide to family assessment and intervention. Philadelphia: FA. Davis; 1994.

5. Bousso RS. Buscando preservar a integridade da unidade familiar: a família vivendo a experiência de ter um filho na UTI pediátrica. [tese]. São Paulo (SP): Escola de Enfermagem/ USP; 1999.

6. Cerveny GMO. A família como modelo desconstruindo a patologia. Campinas (SP): Psy II; 1994.

7. Guimaraes FPM. Indivíduos com diabetes mellitus tipo 2: perfil e prática adotada em relação ao tratamento não medicamentoso. [dissertação]. Ribeirão Preto (SP): Escola de Enfermagem de Ribeirão Preto/USP; 2001.

8. Costa AA, Almeida JS de Neto. Manual de diabetes. 3.ed. São Paulo (SP): Sarvier; 1998.

9. Pedrosa HC, Nery ES, Sena FV, Novaes C, Feldkircher TC, Dias MSO, et al. O desafio do projeto salvando o pé diabético. Terapêutica em Diabetes 1999; 4(19):1-10.

10. Souza CR, Zanetti ML. A prática de utilização de seringas descartáveis na administração de insulina no domicílio. Rev Latino-am Enfermagem 2001; 9(1):39-45.

11. Lessa I, Mendonça GAS, Teixeira MTB. Doenças crônicas não transmissíveis no Brasil: dos fatores de risco ao impacto social. Bol Of Sanit Paname 1996; 120(5):389-412.

12. Sociedade Brasileira de Diabetes (SBD). Consenso brasileiro de conceitos e condutas para o diabetes mellitus recomendações da Sociedade Brasileira de Diabetes para a prática clinica - 1997. São Paulo: SBD; 1997.

13. Hass LB. Cronic complication of diabetes mellitus. Nurs Clin North Am 1993; 28(1):71-85.

14. Reiber GE, Lipsky BA, Gibbons GM. The burdem of diabetic foot ulcers. Am J Surg 1998; 176 (Suppl 2):5-10.

15. Levin NE. Foot lesions in patients with diabetes mellitus. Endocrinol Metabolism Clin North Am 1996; 25(2):447-62. 